OPEN ACCESS

Edited by:

Offer Erez,

Soroka Medical Center, Israel

Reviewed by:

Ewa Wender-Ozegowska,

Poznan University of Medical

Sciences, Poland

Antonio Simone Laganà,

University of Insubria, Italy

*Correspondence:

Fanshu Zeng

jian82714@163.com

tThese authors have contributed equally to this work and share first

authorship

Specialty section: This article was submitted to

Neonatology,

a section of the journal

Frontiers in Pediatrics

Received: 24 April 2021

Accepted: 14 June 2021

Published: 09 July 2021

Citation:

Zhong W, Fan X, Hu F, Chen M and Zeng $F$ (2021) Gestational Weight

Gain and Its Effects on Maternal and

Neonatal Outcome in Women With

Twin Pregnancies: A Systematic

Review and Meta-Analysis.

Front. Pediatr. 9:674414

doi: 10.3389/fped.2021.674414

\section{Gestational Weight Gain and Its Effects on Maternal and Neonatal Outcome in Women With Twin Pregnancies: A Systematic Review and Meta-Analysis}

\author{
Wei Zhong ${ }^{1 \dagger}$, Xiaojiao Fan ${ }^{2 \dagger}$, Fang $\mathrm{Hu}^{1}$, Meiqin Chen ${ }^{1}$ and Fanshu Zeng ${ }^{3 *}$ \\ ${ }^{1}$ Department of Obstetrics, Chengdu Traditional Chinese Medicine University Affiliated Hospital, Chengdu, China, \\ ${ }^{2}$ Department of Pediatrics, Chengdu Traditional Chinese Medicine University Affiliated Hospital, Chengdu, China, \\ ${ }^{3}$ Department of Emergency, Chengdu Traditional Chinese Medicine University Affiliated Hospital, Chengdu, China
}

Background: The incidence of twin pregnancies has risen recently. Such pregnancies are associated with an increased risk for poor maternal and infant outcomes. Gestational weight gain, particularly in singleton pregnancies, has been well-linked with maternal and infant outcomes. The aim of the current meta-analysis was to evaluate the effects of gestational weight gain on maternal and fetal outcomes in women with twin pregnancies.

Methods: A systematic search was conducted using the PubMed, Scopus, and Google Scholar databases. Studies, either retrospective or prospective in design, evaluating the effects of gestational weight gain (defined using Institute of Medicine (IOM) guidelines) maternal and/or fetal/neonatal outcomes in women with twin pregnancies were included. Statistical analysis was performed using STATA software.

Results: Eleven studies were included in the meta-analysis. Mothers with inadequate weight gain had increased risk for gestational diabetes mellitus (OR 1.19; 95\% Cl: 1.01, 1.40) and decreased risk for gestational hypertension (OR 0.58; 95\% Cl: 0.49, 0.68) and cesarean section (OR 0.94; 95\% Cl: 0.93, 0.96). Neonates born to mothers with inadequate weight gain were susceptible to increased risk for preterm delivery (OR 1.17; 95\% Cl: 1.03, 1.34), very preterm delivery (gestational age <32 weeks) (OR 1.84; 95\% Cl: 1.36, 2.48), small for gestational age status (OR 1.41; 95\% Cl: 1.15, 1.72), low birth weight status (<2,500 g) (OR 1.27; 95\% Cl: 1.17, 1.38), and neonatal intensive care unit (NICU) admission (OR 1.16; 95\% Cl: 1.08, 1.24). The pooled findings indicate an increased risk for gestational hypertension (OR 1.82; 95\% Cl: 1.60, 2.06) and cesarean section (OR 1.07; 95\% Cl: 1.05, 1.08) among mothers with excessive weight gain. Neonates born to mothers with excessive weight gain were susceptible to increased risk for preterm delivery and very preterm delivery, but were associated with a decreased risk for low birth weight status and small for gestational age status.

Conclusions: Gestational weight gain in twin pregnancy, either lower or higher than IOM recommended guidelines, is associated with poor maternal and neonatal outcomes. Our findings call for incorporating counseling on optimal weight gain during pregnancy as part of routine antenatal visits.

Keywords: gestational weight gain, twin pregnancy, maternal outcomes, neonatal outcomes, meta-analysis 


\section{INTRODUCTION}

The incidence of twin pregnancies has increased over the last 2-3 decades $(1,2)$, and given that twin pregnancies have been linked with increased risk of poor maternal and infant outcomes (3-6), this could potentially emerge as a substantial clinical and public health problem. Furthermore, a wide array of obstetric and perinatal complications are linked to multiple gestation, including hypertensive disorders, gestational diabetes, prematurity, low birth weight, small sizes for gestational age, and perinatal death (3-8). In particular, prematurity is one of the common outcomes of multiple gestation and adds a substantial burden on the health care system $(9,10)$. According to a reliable estimate, while twin pregnancies constitute only around 3-4\% of all birth globally, they contribute to more than one-fifth (20\%) of the burden of preterm births $(11,12)$.

Among a multitude of factors that could potentially impact pregnancy outcomes, one of the readily modifiable factors is gestational weight gain $(13,14)$. The Institute of Medicine (IOM) has developed recommendations for singleton and twin pregnancies, determining optimum weight gain on the basis of the mother's pre-pregnancy body mass index (BMI) (15, 16). For the twin pregnancies, the IOM guidelines suggest the optimal weight gain to be $16.8-24.5 \mathrm{~kg}(37-54 \mathrm{lb})$ or $0.45-0.66$ $\mathrm{kg} /$ weeks for normal pre-pregnancy BMI (18.5-24.9 kg/m²), $14.1-22.7 \mathrm{~kg}(31-50 \mathrm{lb})$ or $0.38-0.61 \mathrm{~kg} /$ weeks for overweight BMI $\left(25-29.9 \mathrm{~kg} / \mathrm{m}^{2}\right)$ and $11.3-19.1 \mathrm{~kg}(25-42 \mathrm{lb})$ or $0.31-$ $0.52 \mathrm{~kg} /$ weeks for obese BMI $\left(\geq 30 \mathrm{~kg} / \mathrm{m}^{2}\right)$ mothers $(15,16)$. A recently published meta-analysis using 23 studies documented an increased risk of small for gestational age (SGA) status and preterm birth in mothers with inadequate gestational weight gain (13). Furthermore, the review noted a $34 \%$ lower risk of SGA and $23 \%$ lower risk of preterm birth in mothers with excessive gestational weight gain (13), although this was countered by an increased risk of cesarean delivery.

Despite this work and the guidelines that have been established as a result, various gaps in our understanding still require further research. To date, several studies have analyzed the influence of high and low gestational weight gain (relative to IOM guidelines) in women with twin pregnancies. However, no study has attempted to pool and analyze this evidence. Thus, the aim of the current study was to conduct a meta-analysis to evaluate maternal and fetal outcomes in women with twin pregnancies depending on gestational weight gain.

\section{MATERIALS AND METHODS}

\section{Search Strategy}

This meta-analysis was conducted according to Preferred Reporting Items for Systematic Reviews and Meta-Analyses (PRISMA) guidelines. Three academic databases (PubMed, Scopus, and Google Scholar) were systematically searched for English language publications made available prior to 31st January 2021. The search strategy included medical topic heading (MeSH) terminology and free text words (Supplementary Table 1). Studies involving twin pregnancies that evaluated maternal and/or fetal/neonatal outcomes based on gestational weight gain were marked for potential inclusion. Reference sections for included studies were also screened for additional potential study candidates.

\section{Selection Criteria and Methods}

The literature screen was conducted by two experts. Potential candidate articles were first examined to remove duplicates, following which full texts were reviewed and evaluated. Disputes concerning study inclusion were resolved via discussion between all study authors.

\section{Inclusion Criteria}

Studies must have been either retrospective record-based or prospective in design to be considered for inclusion. Included studies must have examined the relationship between maternal gestational weight gain during pregnancy and maternal and fetal/neonatal outcomes of interest such as gestational diabetes mellitus, gestational hypertension/pre-eclampsia, premature rupture of membranes, cesarean delivery, postpartum hemorrhage, preterm delivery, small for gestational age state, low birth weight, and admission to neonatal intensive care unit (NICU).

\section{Exclusion Criteria}

Case-reports or review articles were excluded. Also, studies that did not provide data on the above listed outcomes of interest were excluded.

\section{Data Extraction and Quality Assessment}

Data from included studies was extracted and compiled by two separate individuals. The following data was extracted: study identification information, study setting, design characteristics, operational definition of adequate gestational weight gain, sample size, comparison groups, and main findings. The quality of all included studies was assessed using the Newcastle-Ottawa Quality Assessment Scale (17).

\section{Statistical Analysis}

This meta-analysis was conducted using STATA software (version 13.0). Effect sizes were reported as pooled odds ratios with 95\% confidence intervals (CIs) for categorical outcomes. For continuous outcomes, effect sizes were reported as weighted mean differences (WMDs). Since the effect of gestational weight gain on birth outcomes was affected by maternal pre-pregnancy BMI, subgroup analysis was done based on maternal prepregnancy body mass index. Maternal pre-pregnancy BMI was categorized as normal (18.5-24.9 kg/m²); overweight (25-29.9 $\left.\mathrm{kg} / \mathrm{m}^{2}\right)$, or obese $\left(\mathrm{BMI} \geq 30 \mathrm{~kg} / \mathrm{m}^{2}\right) . I^{2}$ was used as a measure to denote heterogeneity. In instances where $I^{2}$ exceeded $\sim 40 \%$, a random effects model was used (18). For reporting statistical significance, a $p<0.05$ was considered. Egger's test was employed to assess for the presence or absence of publication bias. Bias assessment was further supported by visual inspection of funnel plots. 


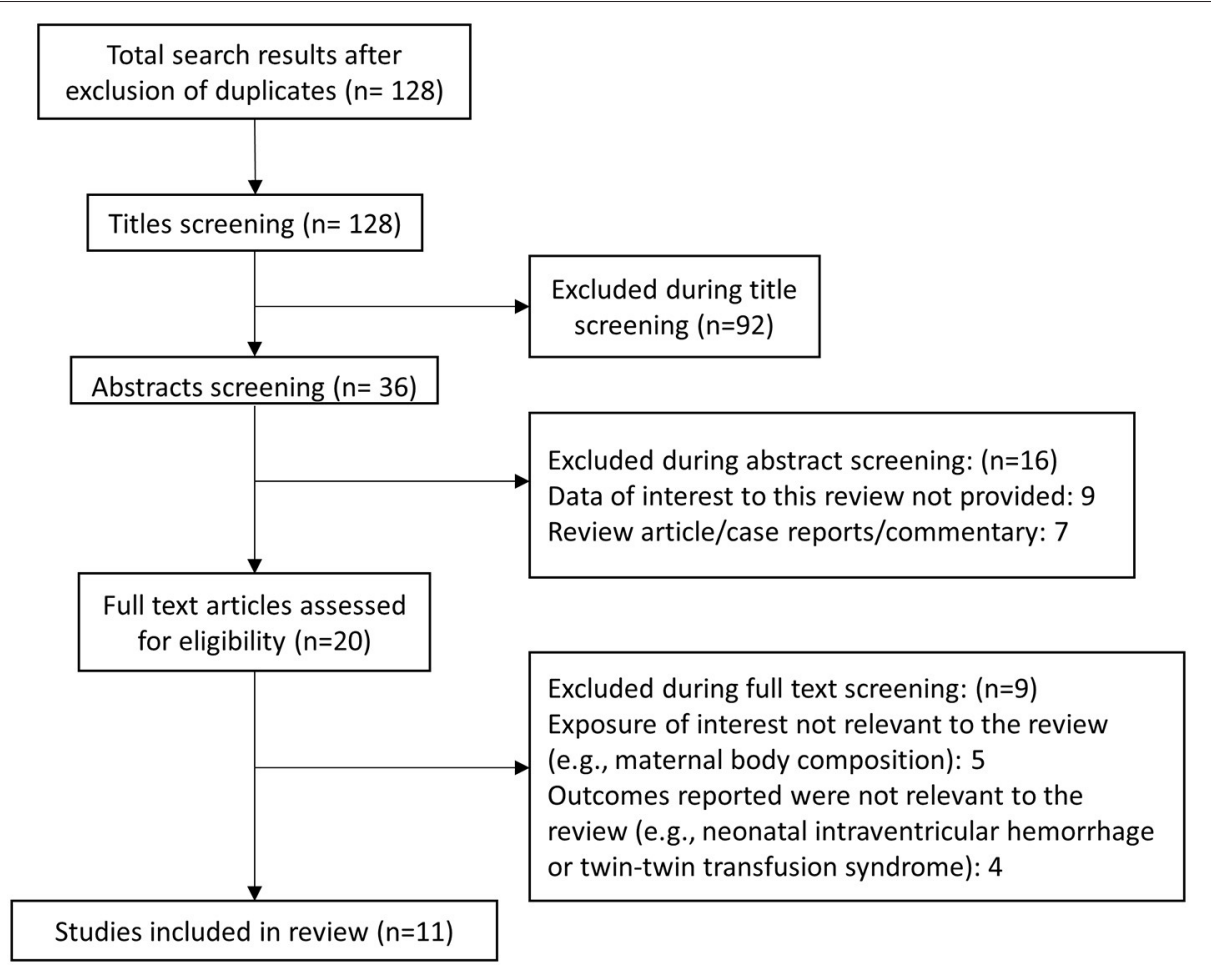

FIGURE 1 | Study inclusion selection process.

\section{RESULTS}

\section{Selection of Articles, Study Characteristics, and Quality of Included Studies}

Literature search revealed 128 unique studies for consideration (Figure 1). Additional screening resulted in the exclusion of 92 studies. Out of the remaining 36 studies, another 16 were excluded after abstract review, while another nine were removed after detailed review. A final total of 11 articles were included in this meta-analysis (Supplementary Table 2). (1929). All included studies used Institute of Medicine (IOM) guidelines to classify adequacy of gestational weight gain according to the pre-pregnancy maternal body mass index (BMI). All included studies performed retrospective analysis of previously collected data. Newcastle-Ottawa Quality Assessment Scale analysis indicated that all included studies were of good quality (Supplementary Table 3).

\section{Overall Pooled Analysis Results Inadequate/Low Weight Gain Compared to Adequate Weight Gain \\ Maternal Outcomes}

Pooled analysis indicated that mothers with inadequate weight gain had increased risk of gestational diabetes mellitus (OR 1.19; 95\% CI: $1.01,1.40 ; I^{2}=0.0 \% ; N=4$ ), compared to those with adequate weight gain (Figure 2). However, those with inadequate weight gain had lower risk of gestational hypertension (OR 0.58; 95\% CI: $0.49,0.68 ; I^{2}=11.7 \% ; N=7$ ) and cesarean section (OR 0.94; 95\% CI: 0.93, 0.96; $I^{2}=0.0 \% ; N=4$ ). Risk of premature membrane rupture (OR 1.09; 95\% CI: 0.91, $\left.1.31 ; I^{2}=0.0 \% ; N=4\right)$ or postpartum hemorrhage (OR 0.96; 95\% CI: $0.73,1.26 ; I^{2}=0.0 \% ; N=2$ ) did not differ significantly between mothers with adequate and inadequate weight gain (Figure 2). There was no evidence of publication bias using Egger's test and a funnel plot (Supplementary Figure 1, $P=0.71)$.

\section{Neonatal Outcomes}

Children born to mothers with inadequate weight gain presented an increased risk of preterm delivery (OR 1.17; 95\% CI: 1.03, 1.34; $I^{2}=72.7 \% ; N=6$ ), very preterm delivery (gestational age $<32$ weeks) (OR 1.84; 95\% CI: $1.36,2.48 ; I^{2}=62.1 \% ; N=6$ ), small for gestational age status (OR 1.41; 95\% CI: $1.15,1.72 ; I^{2}=84.4 \%$; $N=8$ ), low birth weight (birth weight $<2,500$ g) (OR 1.27; 95\% CI: $1.17,1.38 ; I^{2}=63.7 \% ; N=5$ ), and NICU admission (OR 1.16; 95\% CI: $1.08,1.24 ; I^{2}=0.0 \% ; N=4$ ) compared to those born to mothers with adequate weight gain (Figure 3 ). There was no evidence of publication bias using Egger's test and a funnel plot (Supplementary Figure 2, $P=0.43$ ). Neonates born to mothers with inadequate weight gain had lower gestational ages (in weeks) (WMD -0.76; 95\% CI: $-1.20,-0.33 ; I^{2}=93.0 \% ; N=5$ ) and mean birth weights (in grams) (WMD -154.7 ; 95\% CI: -217.1 , 
Author

$\operatorname{RR}(95 \% \mathrm{Cl})$
Events, Events, \%

Treatment Control Weight

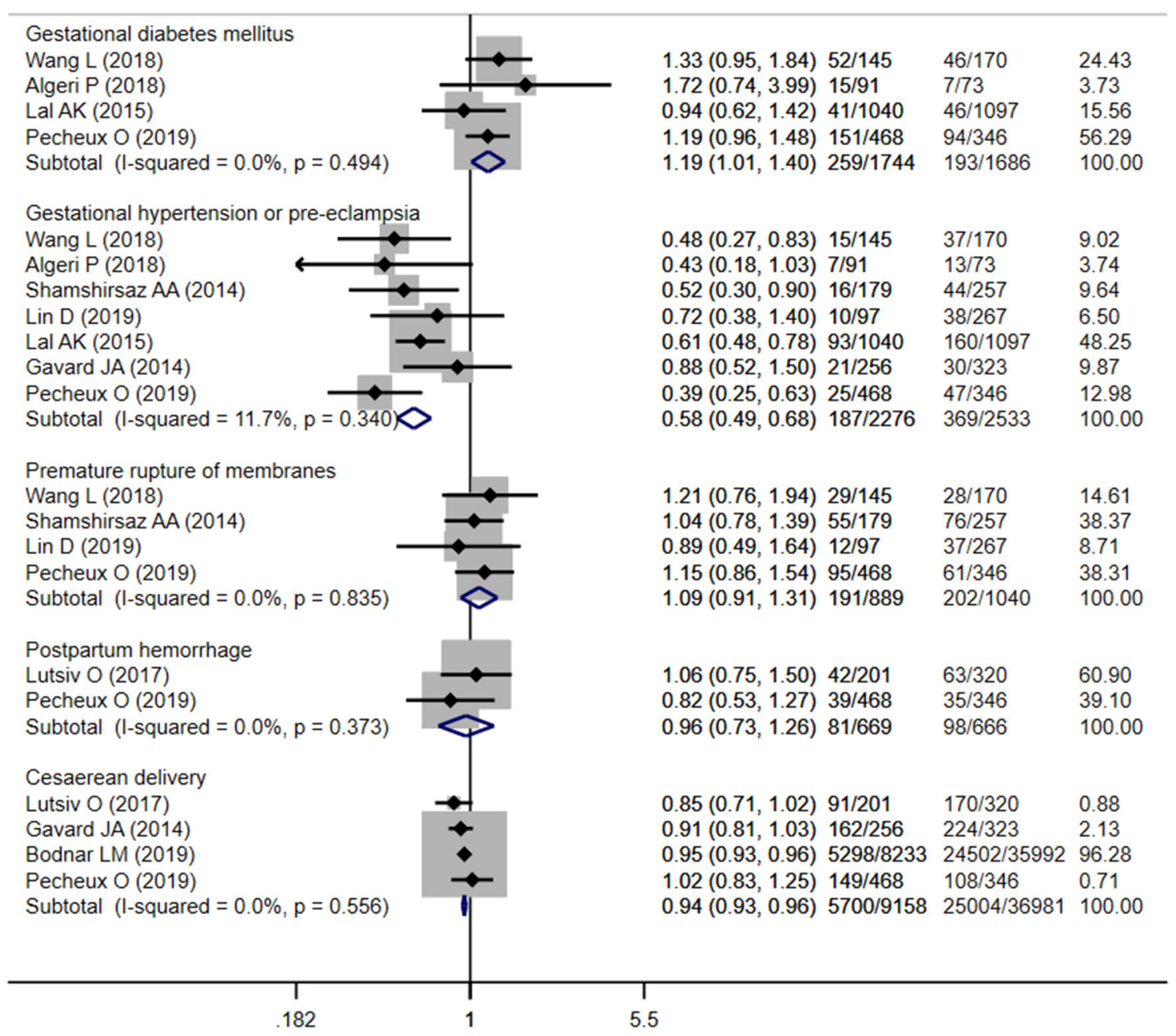

FIGURE 2 | Pooled analysis of inadequate or adequate pregnancy weight gain effects on maternal health outcomes.

-92.2; $\left.I^{2}=92.1 \% ; N=4\right)$ compared to those born to mothers with adequate weight gain (Figures 4, 5).

\section{Excessive Weight Gain Compared to Adequate Weight Gain \\ Maternal Outcomes}

The findings indicated an increased risk of gestational hypertension (OR 1.82; 95\% CI: 1.60, 2.06; $I^{2}=31.3 \%$; $N=8$ ) and cesarean section (OR 1.07; 95\% CI: $1.05,1.08 ; I^{2}=$ $19.7 \% ; N=5$ ) among pregnant mothers with excessive weight gain compared to those with adequate gain (Figure 6). Risk of gestational diabetes mellitus (OR 0.97; 95\% CI: 0.77, 1.24; $I^{2}$ $=8.8 \% ; N=5$ ), premature membrane rupture (OR 0.93; $95 \%$ CI: $\left.0.74,1.17 ; I^{2}=0.0 \% ; N=4\right)$, and postpartum hemorrhage (OR 1.10; 95\% CI: 0.81, 1.49; $I^{2}=27.6 \% ; N=2$ ) did not differ significantly between mothers with adequate and excessive weight gain (Figure 6). There was no evidence of publication bias (Supplementary Figure 3, $P=0.29$ ).

\section{Neonatal Outcomes}

Neonates born to mothers with excessive weight gain were at increased risk of preterm delivery (OR 1.08; 95\% CI: 1.02, 1.14; $I^{2}$ $=51.3 \% ; N=7$ ) and very preterm delivery (gestational age $<32$ weeks) (OR 1.21; 95\% CI: 1.14, 1.28; $I^{2}=0.8 \% ; N=7$ ) (Figure 7). However, in such neonates, the risk of low birth weights (OR 0.87; 95\% CI: $\left.0.82,0.92 ; I^{2}=0.0 \% ; N=6\right)$ and small for gestational age status (OR $0.83 ; 95 \%$ CI: $0.78,0.88 ; I^{2}=37.6 \% ; N=9$ ) was lower compared to children born to mothers with adequate weight gain (Figure 7). There was no evidence of publication bias (Supplementary Figure 4, $P=0.34$ ). Neonates born to mothers with excessive weight gain had similar gestational ages (in weeks) (WMD 0.00; 95\% CI: $-0.17,0.16 ; I^{2}=48.7 \%$; $N=6$ ) compared to those born to mothers with adequate weight gain. While the 
Author

Preterm delivery $(<37$ wks)

Wang L (2018)

Algeri P (2018)

Shamshirsaz AA (2014)

Lutsiv O (2017)

Lin D (2019)

Pecheux O (2019)
Subtotal (I-squared $=72.7 \%, p=0.003)$

Very preterm delivery ( $<32$ wks)

Wang $L$ (2018)

Algeri P (2018)

Shamshirsaz AA (2014)

Lin D (2019)

Bodnar LM (2019)

Pecheux O (2019)

Subtotal $(\mathrm{I}$-squared $=62.1 \%, \mathrm{p}=0.022$

Small for gestational age

Wang L (2018)

Algeri P (2018)

Shamshirsaz AA (2014)

Lutsiv O (2017)

Lin D (2019)

Lal AK (2015)

Bodnar LM (2019)

Pecheux O (2019)

Subtotal (I-squared $=84.4 \%, p=0.000$ )

Low birth weight $(<2500 \mathrm{~g})$

Lutsiv O (2017)

Lin D (2019)

Lal AK (2015)

Gavard JA (2014)

Pecheux O (2019)

Subtotal (I-squared $=63.7 \%, p=0.026)$

Admission to $\mathrm{NICU}$

Lutsiv O (2017)

Lin D (2019)

Lal AK (2015)

Pecheux O (2019)

Subtotal (I-squared $=0.0 \%, p=0.716$ )

NOTE: Weights are from random effects analysis

\begin{tabular}{c|c|c|}
\hline & 1 & 198
\end{tabular}
$\mathrm{RR}(95 \% \mathrm{Cl}) \quad \begin{array}{lll}\text { Events, } & \text { Events, } & \% \\ \text { Treatment } & \text { Control } & \text { Weight }\end{array}$

$0.97(0.81,1.15) \quad 89 / 145$

$1.69(1.23,2.32) 61 / 91$

$1.07(0.98,1.17) \quad 150 / 179$

$1.17(0.97,1.40) 104 / 201$

$1.09(0.96,1.25) 75 / 97$

$1.55(1.18,2.04) 126 / 468$

$1.17(1.03,1.34) 605 / 1181$

$108 / 170$

$29 / 73$

$201 / 257$

$142 / 320$

$189 / 267$

$60 / 346$

$5.86(1.73,19.85) 15 / 145$

$5.86(1.73,19.85) 15 / 145$

$3.69(1.48,9.23) 23 / 91$

$1.82(1.34,2.46) 67 / 179$

$1.15(0.41,3.17) 5 / 97$

$1.42(1.34,1.50) 1318 / 8233$

$1.97(1.17,3.33) 48 / 468$

$1.84(1.36,2.48) \quad 1476 / 9213 \quad 4153 / 37105100.00$

$3 / 170$

$5 / 73$

$53 / 257$

$12 / 267$

10.36

22.50

17.15

12.16

100.00

5.19

8.28

26.39

$4062 / 3599235.85$

$18 / 346 \quad 17.26$

$1.40(0.82,2.39) \quad 25 / 145 \quad 21 / 170 \quad 8.27$

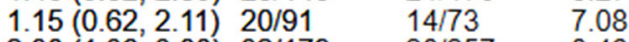

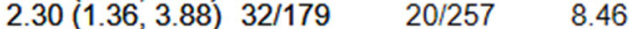

$1.43(1.16,1.76) \quad 121 / 402 \quad 135 / 640 \quad 16.07$

$1.52(0.84,2.73) \quad 16 / 194 \quad 29 / 534 \quad 7.41$

$1.26(1.01,1.57) \quad 149 / 1040 \quad 125 / 1097 \quad 15.78$

$1.68(1.60,1.78) 1552 / 8233 \quad 4030 / 3599219.19$

$1.05(0.91,1.21) \quad 235 / 468 \quad 165 / 346 \quad 17.75$

$1.41(1.15,1.72) 2150 / 107524539 / 39109100.00$

$\begin{array}{llll}1.33(1.16,1.53) & 204 / 402 & 244 / 640 & 17.66 \\ 1.14(1.03,1.26) & 149 / 194 & 360 / 534 & 23.09 \\ 1.34(1.23,1.46) & 595 / 1040 & 469 / 1097 & 24.68 \\ 1.49(1.21,1.84) & 144 / 512 & 122 / 646 & 10.82 \\ 1.21(1.10,1.32) & 362 / 468 & 222 / 346 & 23.74 \\ 1.27(1.17,1.38) & 1454 / 2616 & 1417 / 3263 & 100.00\end{array}$

$1.18(1.01,1.37) \quad 170 / 402 \quad 230 / 640 \quad 20.29$

$1.18(0.97,1.42) 88 / 194 \quad 206 / 534 \quad 13.64$

$1.13(1.03,1.24) 508 / 1040 \quad 474 / 1097 \quad 56.87$

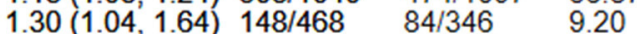

$1.16(1.08,1.24) 914 / 2104 \quad 994 / 2617 \quad 100.00$

FIGURE 3 | Pooled analysis of inadequate or adequate pregnancy weight gain effects on neonatal health outcomes.

pooled mean birth weight (in grams) (WMD 42.3; 95\% CI: - 16.2, $100.8 ; I^{2}=90.3 \% ; N=5$ ) was higher by roughly 42 grams, no statistically significant difference was observed (Figures 8, 9).

\section{Subgroup Analysis Based on Maternal \\ Pre-pregnancy BMI \\ Mothers With Normal Pre-pregnancy BMI}

Maternal inadequate or low gestational weight gain, compared to adequate weight gain, was associated with an increased risk of small for gestational age status (OR 1.62; 95\% CI: 1.30, 2.02; $I^{2}=$ $52.6 \% ; N=5$ ), very preterm status (OR 2.38; 95\% CI: $1.39,4.09$; $\left.I^{2}=76.8 \% ; N=4\right)$, low birth weight (OR 1.25; 95\% CI: 1.15 , $1.37 ; I^{2}=8.3 \% ; N=2$ ), and NICU admission (OR $1.15 ; 95 \% \mathrm{CI}$ :
1.02, 1.30; $N=1$ ) (Supplementary Figure 5). However, the risk of gestational hypertension (OR 0.57; 95\% CI: $0.44,0.73 ; I^{2}=$ $0.0 \% ; N=4$ ) and cesarean delivery (OR 0.93; 95\% CI: 0.91, 0.96; $N=1$ ) was lower (Supplementary Figure 6).

Maternal excessive gestational weight gain, compared to adequate weight gain, was associated with an increased risk of gestational hypertension (OR 1.92; 95\% CI: 1.56, 2.35; $I^{2}=44.4 \% ; N=5$ ), cesarean delivery (OR 1.07; 95\% CI: $1.04,1.09 ; I^{2}=37.6 \% ; N=2$ ), and very preterm delivery (OR 1.20; 95\% CI: $1.10,1.31 ; I^{2}=0.0 \% ; N$ $=5$ ) (Supplementary Figure 7 and Supplementary Figure 8). However, the risk of small for gestational age status was lower (OR 0.80; 95\% CI: 0.72, 0.88; $I^{2}=0.0 \% ; N=5$ ). The risk 


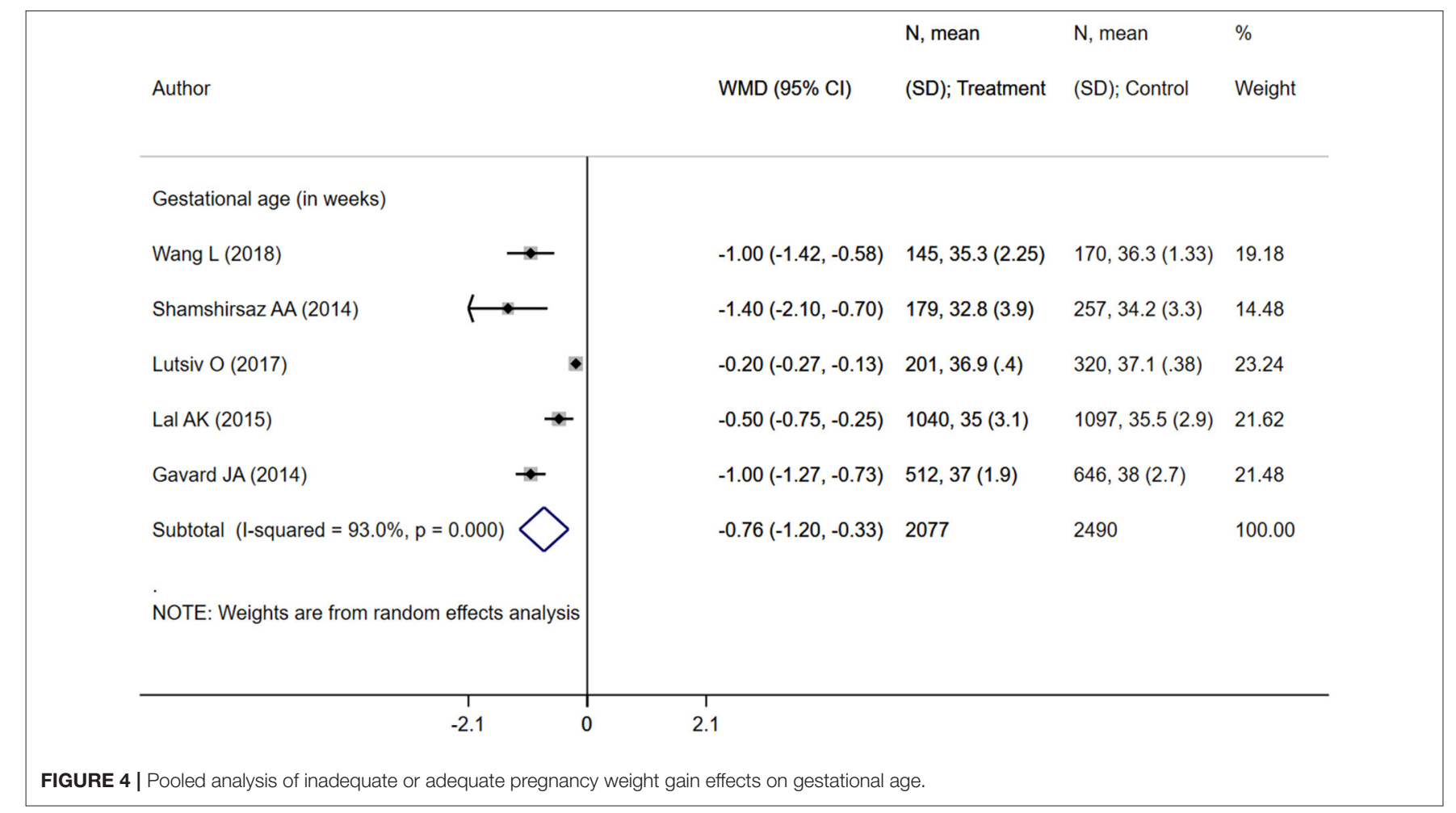

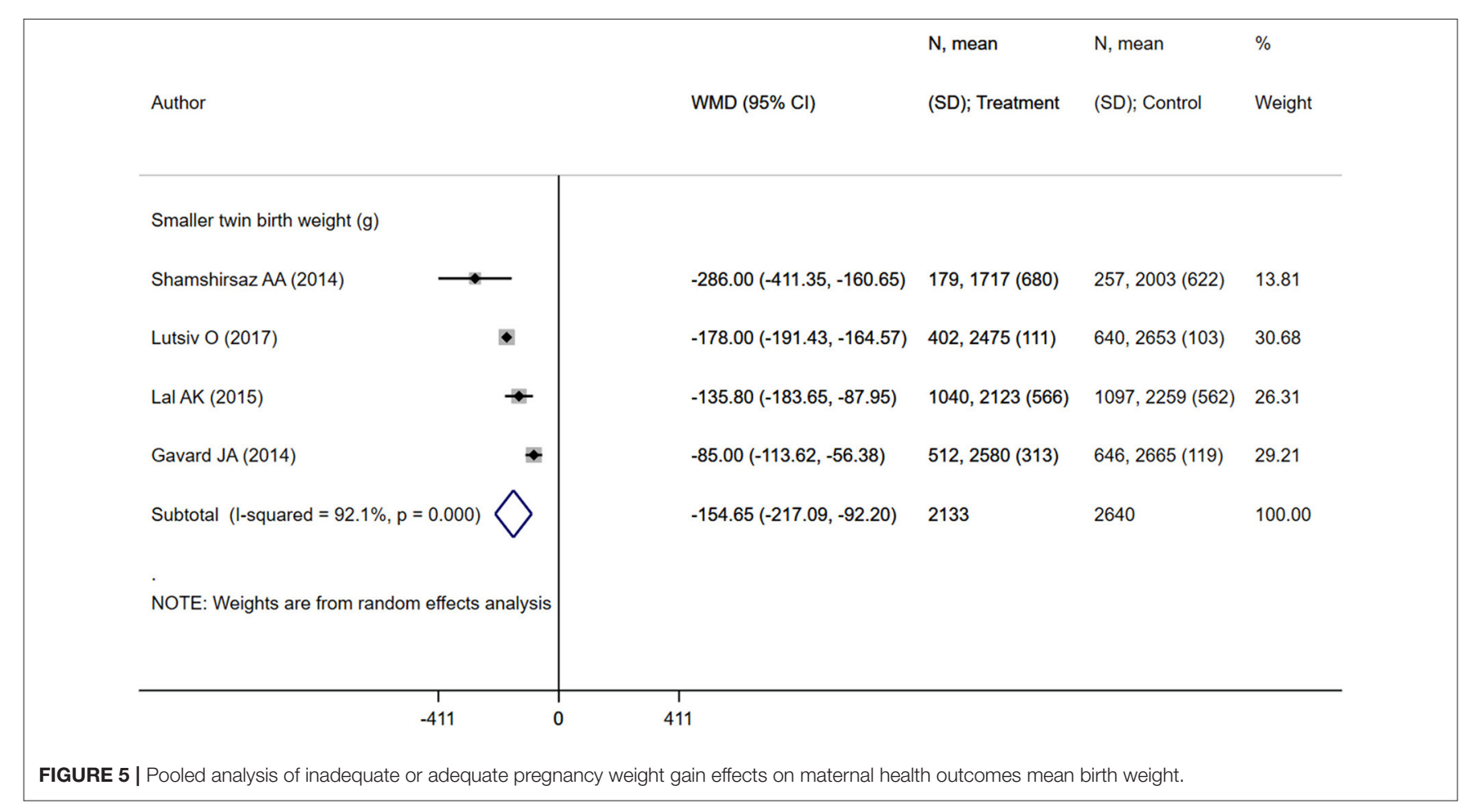

of gestational diabetes mellitus, premature membrane rupture, preterm delivery, low birth weight, and NICU admission did not differ significantly between those with adequate and excessive weight gain.
Overweight Mothers (Pre-pregnancy BMI: 25-29.9 KG/M²) Maternal inadequate or low gestational weight gain, compared to adequate weight gain, was associated with an increased risk of small for gestational age status (OR 1.47; 95\% CI: 1.04, 2.06; 
Author

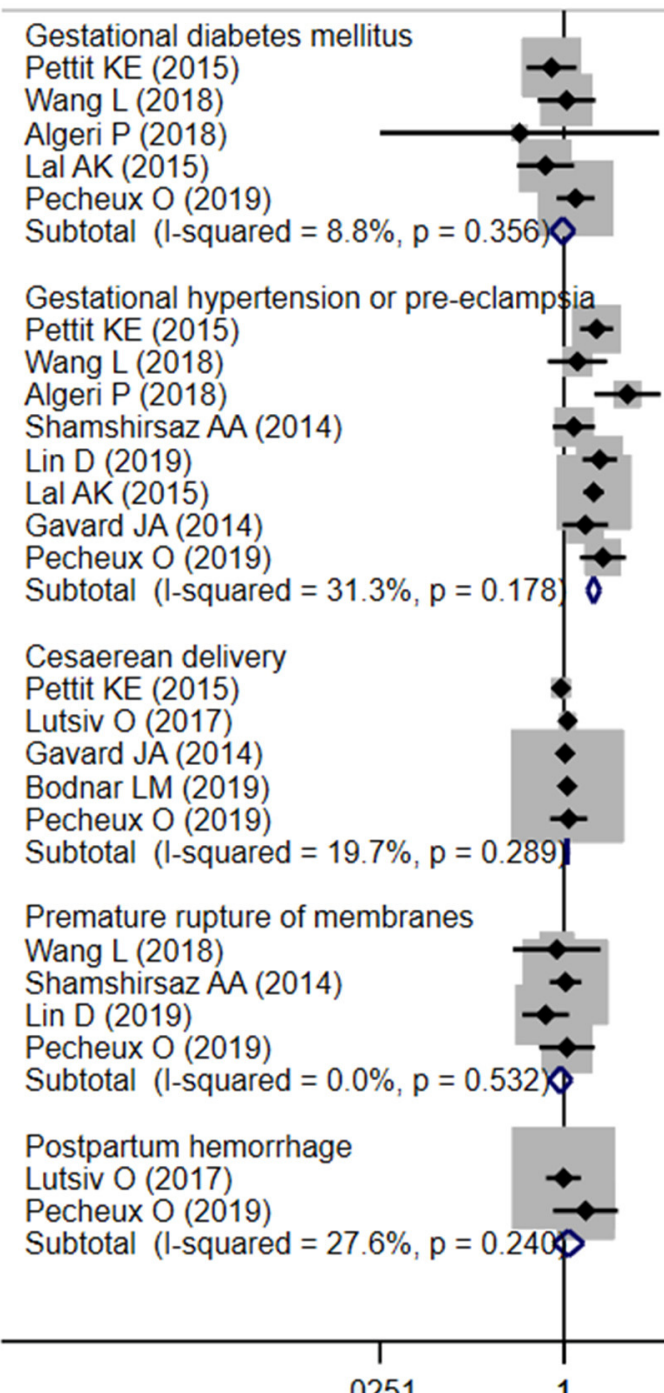

.0251

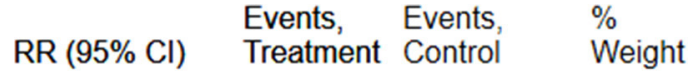

\begin{tabular}{|c|c|}
\hline $\begin{array}{l}0.78(0.47,1.29) 24 / 203 \\
1.06(0.59,1.88) 10 / 35 \\
0.41(0.03,6.74) 0 / 11 \\
0.69(0.39,1.23) 15 / 517 \\
1.27(0.87,1.85) 22 / 64\end{array}$ & $\begin{array}{l}30 / 198 \\
46 / 170 \\
7 / 73 \\
46 / 1097 \\
94 / 346\end{array}$ \\
\hline
\end{tabular}

$0.97(0.77,1.24) 71 / 830 \quad 223 / 1884 \quad 100.00$

$1.93(1.38,2.68) 77 / 203 \quad 39 / 198 \quad 14.69$

$1.31(0.72,2.38) 10 / 35 \quad 37 / 170 \quad 4.55$

$3.57(1.84,6.95) 7 / 11 \quad 13 / 73 \quad 3.65$

$1.22(0.80,1.87) 28 / 134 \quad 44 / 257 \quad 8.94$

$\begin{array}{lll}2.05(1.45,2.90) 82 / 281 & 38 / 267 & 13.48\end{array}$

$\begin{array}{lll}1.82(1.48,2.23) 137 / 517 & 160 / 1097 & 39.31\end{array}$

$1.54(0.98,2.43) 36 / 252 \quad 30 / 323 \quad 7.79$

$2.19(1.38,3.47) 19 / 64 \quad 47 / 346 \quad 7.60$

$1.82(1.60,2.06) 396 / 1497 \quad 408 / 2731 \quad 100.00$

$0.94(0.84,1.06) 146 / 203 \quad 151 / 198 \quad 1.53$

$1.08(0.92,1.26) 126 / 220 \quad 170 / 320 \quad 0.87$

$1.02(0.92,1.14) 179 / 252 \quad 224 / 323 \quad 1.79$

$1.07(1.05,1.08) 6368 / 8754 \quad 24502 / 3599295.67$

$1.10(0.76,1.60) 22 / 64 \quad 108 / 346 \quad 0.15$

$1.07(1.05,1.08) 6841 / 949325155 / 37179100.00$

$0.87(0.36,2.09) 5 / 35 \quad 28 / 170 \quad 6.80$

$1.03(0.75,1.42) 41 / 134 \quad 76 / 257 \quad 52.24$

$0.69(0.43,1.11) 27 / 281 \quad 37 / 267 \quad 24.11$

$1.06(0.61,1.86) 12 / 64 \quad 61 / 346 \quad 16.85$

$0.93(0.74,1.17) 85 / 514 \quad 202 / 1040 \quad 100.00$

$0.99(0.70,1.41) 43 / 220 \quad 63 / 320 \quad 77.77$

$1.54(0.81,2.96) 10 / 64 \quad 35 / 346 \quad 22.23$

$1.10(0.81,1.49) 53 / 284 \quad 98 / 666 \quad 100.00$

FIGURE 6 | Pooled analysis of excessive or adequate pregnancy weight gain effects on maternal health outcomes.

$I^{2}=44.8 \% ; N=3$ ) and very preterm status (OR 1.47; 95\% CI: $1.31,1.65 ; I^{2}=0.0 \% ; N=2$ ) (Supplementary Figure 9). There was a non-statistically significant lower risk of cesarean delivery in mothers with inadequate or low weight gain (OR 0.96; 95\% CI: 0.93, 1.00; $N=1$ ) (Supplementary Figure 10). The risk of gestational diabetes mellitus, hypertension, premature membrane rupture, preterm delivery, low birth weight, and NICU admission did not differ significantly between those with adequate and inadequate/low gestational weight gain.

Maternal excessive gestational weight gain, compared to adequate weight gain, was associated with increased risk of gestational hypertension (OR 1.65; 95\% CI: 1.18, 2.30; $I^{2}=$ $0.0 \% ; N=3$ ) and very preterm delivery (OR 1.24; $95 \% \mathrm{CI}$ : $1.10,1.39 ; I^{2}=0.0 \% ; N=3$ ) (Supplementary Figure 11,
Supplementary Figure 12). However, the risk of gestational diabetes mellitus reduced (OR 0.21; 95\% CI: 0.07, 0.58; $I^{2}=$ $0.0 \% ; N=2)$. The risk of cesarean delivery, premature membrane rupture, preterm delivery, low birth weight, small for gestational age status, and NICU admission was not statistically different between those with adequate and excessive weight gain.

\section{Obese Mothers (Pre-pregnancy BMI: $\geq 30 \mathrm{KG} / \mathrm{M}^{2}$ )}

Maternal inadequate or low gestational weight gain, compared to adequate weight gain, was associated with an increased risk of low birth weight (OR 1.33; 95\% CI: $1.06,1.66 ; I^{2}=70.9 \%$; $N=3$ ), small for gestational age status (OR 1.41; $95 \%$ CI: 1.16, $1.71 ; I^{2}=7.6 \% ; N=3$ ), and very preterm baby (OR 1.32; $95 \%$ CI: $1.14,1.52 ; I^{2}=2.1 \% ; N=2$ ) (Supplementary Figure 13). 


\begin{tabular}{|c|c|c|c|c|}
\hline Author & $\mathrm{RR}(95 \% \mathrm{Cl})$ & $\begin{array}{l}\text { Events, } \\
\text { Treatment }\end{array}$ & $\begin{array}{l}\text { Events, } \\
\text { Control }\end{array}$ & $\begin{array}{l}\% \\
\text { Weight }\end{array}$ \\
\hline $\begin{array}{l}\text { Preterm delivery (<37 wks) } \\
\text { Pettit KE (2015) } \\
\text { Wang L (2018) } \\
\text { Algeri P (2018) } \\
\text { Shamshirsaz AA (2014) } \\
\text { Lutsiv O }(2017) \\
\text { Lin D }(2019) \\
\text { Pecheux O (2019) } \\
\text { Subtotal (I-squared }=51.3 \%, p=0.055)\end{array}$ & $\begin{array}{l}1.02(0.89,1.18) \\
0.99(0.75,1.31) \\
2.06(1.38,3.06) \\
1.09(0.99,1.20) \\
1.10(0.91,1.32) \\
1.05(0.94,1.16) \\
1.35(0.82,2.23) \\
1.08(1.02,1.14)\end{array}$ & $\begin{array}{l}134 / 203 \\
22 / 35 \\
9 / 11 \\
114 / 134 \\
107 / 220 \\
208 / 281 \\
15 / 64 \\
609 / 948\end{array}$ & $\begin{array}{l}128 / 198 \\
108 / 170 \\
29 / 73 \\
201 / 257 \\
142 / 320 \\
189 / 267 \\
60 / 346 \\
857 / 1631\end{array}$ & $\begin{array}{l}16.15 \\
4.22 \\
2.09 \\
35.76 \\
9.82 \\
30.63 \\
1.32 \\
100.00\end{array}$ \\
\hline $\begin{array}{l}\text { Very preterm delivery }(<32 \text { wks) } \\
\text { Pettit KE }(2015) \\
\text { Wang L (2018) } \\
\text { Algeri P (2018) } \\
\text { Shamshirsaz AA (2014) } \\
\text { Lin D (2019) } \\
\text { Bodnar LM (2019) } \\
\text { Pecheux O (2019) } \\
\text { Subtotal (I-squared }=0.8 \%, p=0.418 \text { ) }\end{array}$ & $\begin{array}{l}1.07(0.69,1.65) \\
0.68(0.04,12.85) \\
3.98(1.10,14.37) \\
1.05(0.70,1.57) \\
0.79(0.35,1.80) \\
1.21(1.14,1.29) \\
1.80(0.74,4.36) \\
1.21(1.14,1.28)\end{array}$ & $\begin{array}{l}35 / 203 \\
0 / 35 \\
3 / 11 \\
29 / 134 \\
10 / 281 \\
1196 / 8754 \\
6 / 64 \\
1279 / 9482\end{array}$ & $\begin{array}{l}32 / 198 \\
3 / 170 \\
5 / 73 \\
53 / 257 \\
12 / 267 \\
4062 / 35992 \\
18 / 346 \\
4185 / 37303\end{array}$ & $\begin{array}{l}1.79 \\
0.04 \\
0.21 \\
2.12 \\
0.51 \\
94.89 \\
0.44 \\
100.00\end{array}$ \\
\hline $\begin{array}{l}\text { Low birth weight }(<2500 \mathrm{~g}) \\
\text { Pettit KE }(2015) \\
\text { Lutsiv O }(2017) \\
\text { Lin D }(2019) \\
\text { Lal AK }(2015) \\
\text { Gavard JA }(2014) \\
\text { Pecheux O (2019) } \\
\text { Subtotal (I-squared }=0.0 \%, p=0.624)\end{array}$ & $\begin{array}{l}0.89(0.78,1.01) \\
0.92(0.79,1.08) \\
0.83(0.75,0.91) \\
0.83(0.72,0.95) \\
0.91(0.71,1.17) \\
0.97(0.79,1.20) \\
0.87(0.82,0.92)\end{array}$ & $\begin{array}{l}133 / 201 \\
155 / 440 \\
314 / 562 \\
183 / 517 \\
87 / 504 \\
40 / 64 \\
912 / 2288\end{array}$ & $\begin{array}{l}145 / 195 \\
244 / 640 \\
360 / 534 \\
469 / 1097 \\
122 / 646 \\
222 / 346 \\
1562 / 3458\end{array}$ & $\begin{array}{l}19.70 \\
12.65 \\
36.77 \\
17.88 \\
5.26 \\
7.73 \\
100.00\end{array}$ \\
\hline $\begin{array}{l}\text { Small for gestational age } \\
\text { Pettit KE (2015) } \\
\text { Wang L (2018) } \\
\text { Algeri P (2018) } \\
\text { Shamshirsaz AA (2014) } \\
\text { Lutsiv O }(2017) \\
\text { Lin D }(2019) \\
\text { Lal AK }(2015) \\
\text { Bodnar LM (2019) } \\
\text { Pecheux O (2019) } \\
\text { Subtotal (I-squared }=37.6 \%, p=0.118)\end{array}$ & $\begin{array}{l}0.60(0.43,0.82) \\
0.46(0.11,1.88) \\
0.21(0.01,3.33) \\
1.63(0.88,3.01) \\
0.94(0.74,1.19) \\
0.79(0.46,1.33) \\
0.71(0.51,1.00) \\
0.84(0.78,0.90) \\
0.72(0.50,1.03) \\
0.83(0.78,0.88)\end{array}$ & $\begin{array}{l}43 / 201 \\
2 / 35 \\
0 / 11 \\
17 / 134 \\
87 / 440 \\
24 / 562 \\
42 / 517 \\
824 / 8754 \\
22 / 64 \\
1061 / 10718\end{array}$ & $\begin{array}{l}70 / 195 \\
21 / 170 \\
14 / 73 \\
20 / 257 \\
135 / 640 \\
29 / 534 \\
125 / 1097 \\
4030 / 35992 \\
165 / 346 \\
4609 / 39304\end{array}$ & $\begin{array}{l}3.83 \\
0.20 \\
0.05 \\
1.08 \\
6.97 \\
1.45 \\
3.63 \\
79.60 \\
3.18 \\
100.00\end{array}$ \\
\hline $\begin{array}{l}\text { Admission to NICU } \\
\text { Pettit KE (2015) } \\
\text { Lutsiv O }(2017) \\
\text { Lin D (2019) } \\
\text { Lal AK }(2015) \\
\text { Pecheux O (2019) } \\
\text { Subtotal (I-squared }=0.0 \%, p=0.455)\end{array}$ & $\begin{array}{l}0.94(0.86,1.03) \\
0.98(0.83,1.15) \\
0.90(0.77,1.06) \\
1.02(0.90,1.14) \\
1.29(0.86,1.94) \\
0.97(0.91,1.02)\end{array}$ & $\begin{array}{l}162 / 201 \\
155 / 440 \\
196 / 562 \\
227 / 517 \\
20 / 64 \\
760 / 1784\end{array}$ & $\begin{array}{l}167 / 195 \\
230 / 640 \\
206 / 534 \\
474 / 1097 \\
84 / 346 \\
1161 / 2812\end{array}$ & $\begin{array}{l}44.84 \\
13.25 \\
14.64 \\
25.15 \\
2.13 \\
100.00\end{array}$ \\
\hline .0136 & & & & \\
\hline
\end{tabular}

FIGURE 7 | Pooled analysis of excessive or adequate pregnancy weight gain effects on neonatal health outcomes.

There was a reduced risk of cesarean delivery (OR 0.95; 95\% CI: $0.93,0.98 ; I^{2}=0.0 \% ; N=2$ ) and gestational hypertension (OR $0.62 ; 95 \%$ CI: $0.44,0.87 ; I^{2}=30.1 \% ; N=3$ ) in mothers with inadequate or low weight gain (Supplementary Figure 14). The risk of gestational diabetes mellitus, premature membrane rupture, preterm delivery, and NICU admission did not differ significantly between those with adequate and inadequate/low gestational weight gain.

Maternal excessive gestational weight gain, compared to adequate weight gain, was associated with an increased risk of gestational hypertension (OR 1.45; 95\% CI: 1.13, $1.86 ; I^{2}=$ $0.0 \% ; N=4$ ), cesarean delivery (OR 1.07; 95\% CI: 1.05, 1.10; $I^{2}=0.0 \% ; N=3$ ), and very preterm delivery (OR $1.20 ; 95 \%$ CI: $1.06,1.34 ; I^{2}=54.8 \% ; N=3$ ) (Supplementary Figure 15, Supplementary Figure 16). The risk of small for gestational age status was reduced (OR 0.85; 95\% CI: 0.74, 0.98; $I^{2}=$ $21.5 \% ; N=3$ ). The risk of premature membrane rupture, gestational diabetes, preterm delivery, low birth weight, and NICU admission did not differ significantly between those with adequate and excessive weight gain. 


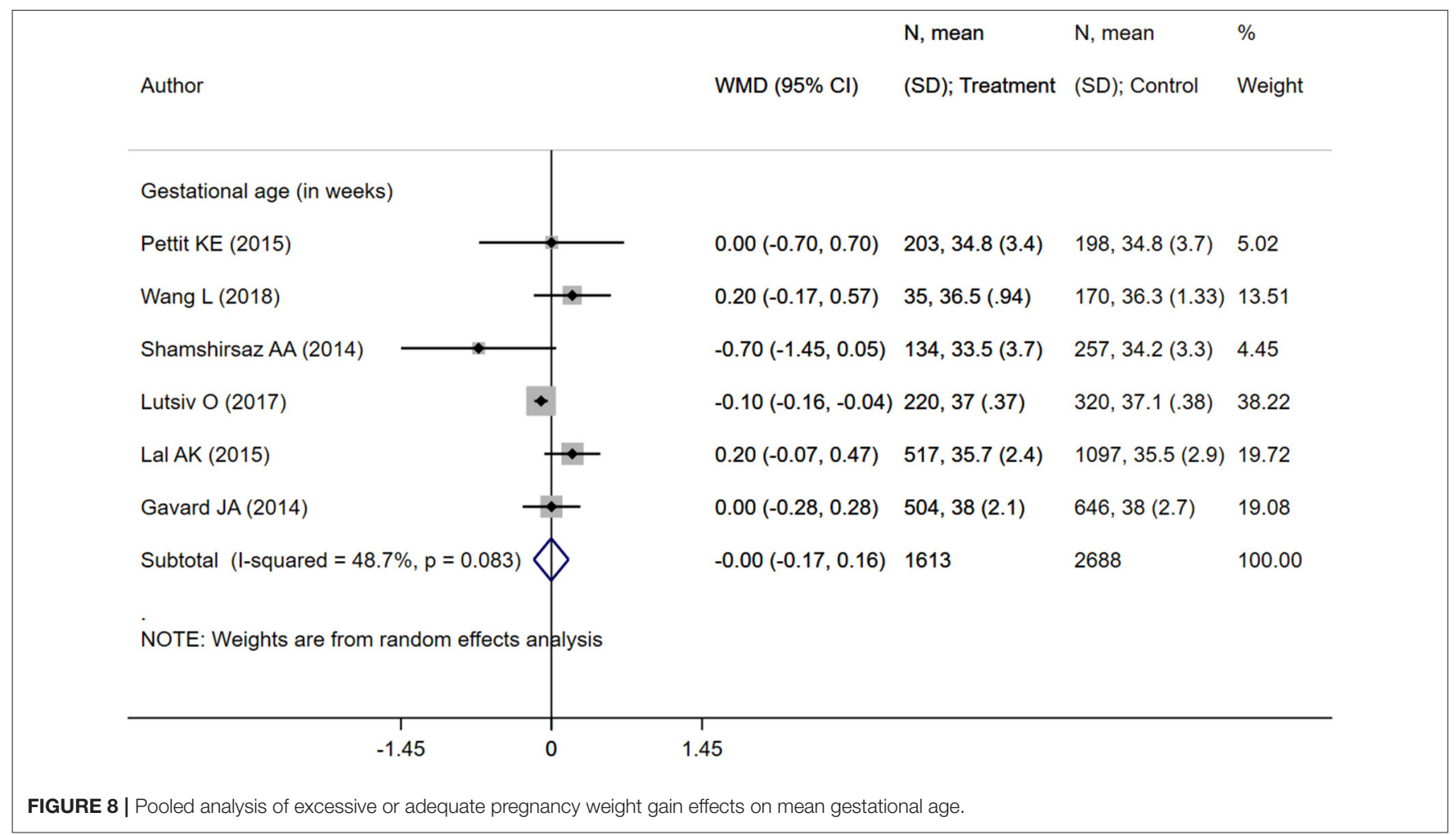

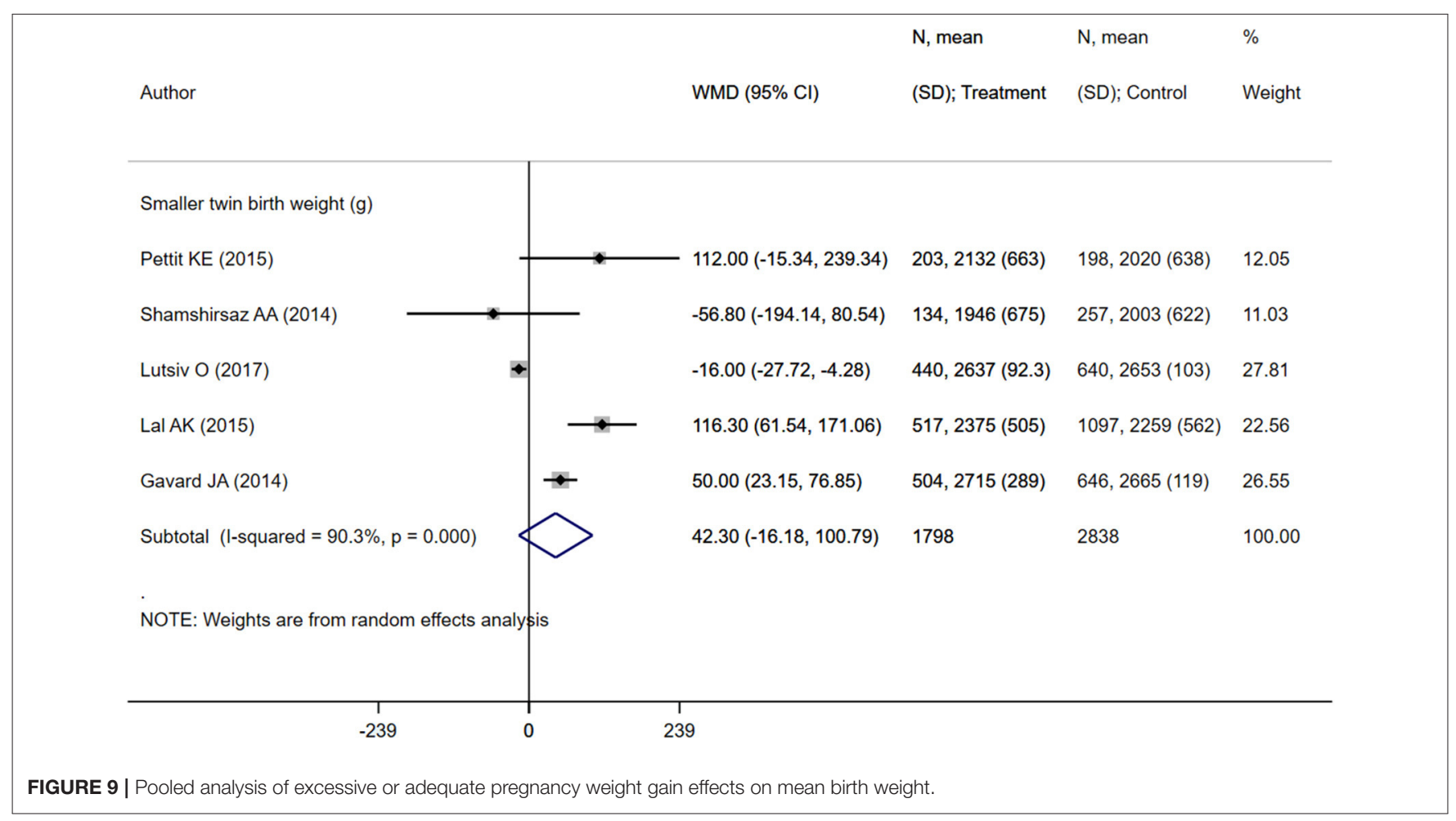




\section{DISCUSSION}

This meta-analysis aimed to evaluate the effects of gestational weight gain on maternal and fetal outcomes in women with twin pregnancies. We herein found an increased risk of negative maternal and fetal outcomes in women experiencing either inadequate or excessive weight gain relative to mothers with optimal gestational weight gain. Mothers with inadequate weight gain were at increased risk for gestational diabetes mellitus, preterm delivery, very preterm delivery, small for gestational age status, low birth weights, and NICU admission. The study also noted an increased risk for gestational hypertension and cesarean section among mothers with excessive weight gain. Neonates born to mothers with excessive weight gain were at increased risk for preterm delivery and very preterm delivery. The findings were largely the same when analysis was conducted based on maternal pre-pregnancy BMI.

A similar meta-analysis, containing 23 studies, was previously conducted for singleton pregnancies (13), finding a higher risk for small for gestational age status and preterm birth when gestational weight gain was below recommended amounts. Moreover, gestational weight gain above baseline was associated with decreased risk for SGA and preterm birth, but increased risk for cesarean delivery (13). Additionally, a large populationbased cohort study in United States found that mothers with low gestational weight gain presented increased risk for serious adverse birth effects including maternal and perinatal death (30). The American study also noted an increased risk for poor maternal and fetal outcomes in mothers with excessive gestational weight gain-however, this increased risk was not found in maternal overweight at the time of conception (30).

Low pregnancy weight gain usually indicates the presence of nutritional deficiencies, inadequate expansion of plasma volume and an underlying metabolic state that is supportive of increased risk of infections and inflammation $(31,32)$. Similarly, it is welldocumented that overweight and obesity is a pro-inflammatory state and excessive weight gain during pregnancy is associated with surge in pro-inflammatory cytokines (33-35). All these factors increase the risk of preterm and very preterm delivery. Also, inflammation leads to activation and subsequent expression of several proteins, cytokines and interleukins that suppress the insulin signaling pathways, lead to less sensitivity to insulin and consequent insulin resistance (36). These factors could, in some way, explain the increased risk of preterm delivery and gestational diabetes in women with either low or high gestational weight gain.

Together with the present study, these findings call for prenatal and antenatal programs to support maternal nutrition and encourage optimal gestational weight gain. Pre-conception nutrition programs should be devised that target normal BMIs for expectant mothers. These programs are currently lacking in both developed and developing countries. In addition, mothers should be educated about the health advantages of maintaining normal BMI and achieving optimal weight gain during pregnancy. Lifestyle and nutritional interventions may assist in these endeavors. It is also important to note that the current evidence suggests that glucose intolerance during pregnancy increases the likelihood of developing abnormal glucose tolerance and diabetes later during life (37). In this context, it is important to ensure a healthy lifestyle and nutritional habits starting from early in the pregnancy. Additional to that, myo-inositol, one of the promising molecules that has not only shown to positively affect fertility but also prevention of gestational diabetes mellitus, could be used during pregnancy $(38,39)$. In a recent Cochrane review, it was found to reduce the incidence of gestational diabetes mellitus by $10-$ $22 \%$ (40). Myo-inositol functions by participating in several signaling processes involving insulin and gonadotropins (38-40). Furthermore, women developing abnormal glucose tolerance during pregnancy should closely monitor their glucose levels post-delivery as well.

This study does have several limitations. First, the included studies were mostly conducted in developed settings with minimal representation from low/middle-income demographic groups. This may make the findings difficult to generalize. Second, while included studies reported preterm birth data, no clear distinctions between spontaneous and induced preterm birth were included. Studies also did not mention how gestational age was assessed, which may affect prematurityrelated observations. However, the studies reported on small for gestational age based on the standard definition of weight below the 10th percentile for the gestational age (41). The studies did not demarcate elective and emergency cesarean deliveries. Most of the studies included in this review were from developed settings and as part of the standard care in these settings, twin pregnancies are usually delivered through elective cesarean section. Therefore, the observed pooled effect sizes for cesarean section as an outcome may be less reliable. Finally, the findings from this meta-analysis are based on observational data and therefore may not be suitable for establishing causality. Our meta-analysis is limited by the use of studies involving retrospective data procurement and analysis, meaning that data on important confounding factors are not always available. Consequently, the observed associations reported by individual studies may not be accurate, thereby affecting the reliability of the findings of this present meta-analysis.

One important aspect to acknowledge is that the IOM guidelines for adequate weight gain lay a wide range and it is often confusing to decide on what ideal minimum weight gain to aim for. Previously conducted studies, especially in overweight and obese women, have shown that weight gain below the IOM recommendations seemingly does not have any significant negative effect of maternal and neonatal outcomes $(14,42,43)$. This may indicate that fetal growth is more important to assess, as opposed to maternal weight gain. Future studies should aim to answer the question of minimum optimal pregnancy weight gain in twin pregnancies across various maternal pre-pregnancy BMI categories.

The findings of the meta-analysis place emphasis on the importance of attaining optimal gestational weight gain in mothers for twin pregnancies. Doing so 
would possibly aid in preventing adverse maternal and fetal/neonatal outcomes. Counseling on optimal weight gain during pregnancy as part of routine antenatal visits is therefore desirable.

\section{DATA AVAILABILITY STATEMENT}

The original contributions presented in the study are included in the article/Supplementary Materials, further inquiries can be directed to the corresponding author/s.

\section{REFERENCES}

1. Collins J. Global epidemiology of multiple birth. Reprod Biomed Online. (2007) 15 (Suppl. 3):45-52. doi: 10.1016/S1472-6483(10)62251-1

2. Smits J, Monden C. Twinning across the developing world. PLoS ONE. (2011) 6:e25239. doi: 10.1371/journal.pone.0025239

3. Walker MC, Murphy KE, Pan S, Yang Q, Wen SW. Adverse maternal outcomes in multifetal pregnancies. BJOG. (2004) 111:1294-6. doi: 10.1111/j.1471-0528.2004.00345.x

4. Vogel JP, Torloni MR, Seuc A, Betrán AP, Widmer M, Souza JP, et al. Maternal and perinatal outcomes of twin pregnancy in 23 low- and middle-income countries. PLoS ONE. (2013) 8:e70549. doi: 10.1371/journal.pone.0070549

5. Rao A, Sairam S, Shehata H. Obstetric complications of twin pregnancies. Best Pract Res Clin Obstet Gynaecol. (2004) 18:557-76. doi: 10.1016/j.bpobgyn.2004.04.007

6. Gebremedhin S. Multiple births in sub-saharan africa: epidemiology, postnatal survival, and growth pattern. Twin Res Hum Gen. (2015) 18:100-7. doi: $10.1017 /$ thg.2014.82

7. Chowdhury S, Hussain MA. Maternal complications in twin pregnancies. Mymensingh Med J. (2011) 20:83-7.

8. Cheong-See F, Schuit E, Arroyo-Manzano D, Khalil A, Barrett J, Joseph KS, et al. Prospective risk of stillbirth and neonatal complications in twin pregnancies: systematic review and meta-analysis. BMJ. (2016) 354:i4353. doi: 10.1136/bmj.i4353

9. Bornstein E, Proudfit CL, Keeler SM. Prematurity in twin pregnancies. Minerva Ginecol. (2009) 61:113-26.

10. Kurdi AM, Mesleh RA, Al-Hakeem MM, Khashoggi TY, Khalifa HM. Multiple pregnancy and preterm labor. Saudi Med J. (2004) 25:632-7.

11. National Institute for Health and Care Excellence. Multiple Pregnancy: Antenatal Care for Twin and Triplet Pregnancies. Clinical Guideline no. 129. London: NICE (2011). Available online at: https://www.nice.org.uk/guidance/ cg129

12. Fuchs F, Senat MV. Multiple gestations and preterm birth. Semin Fetal Neonatal Med. (2016) 21:113-20. doi: 10.1016/j.siny.2015.12.010

13. Goldstein RF, Abell SK, Ranasinha S, Misso M, Boyle JA, Black MH, et al. Association of gestational weight gain with maternal and infant outcomes: a systematic review and meta-analysis. JAMA. (2017) 317:220725. doi: 10.1001/jama.2017.3635

14. Oken E, Kleinman KP, Belfort MB, Hammitt JK, Gillman MW. Associations of gestational weight gain with short- and longer-term maternal and child health outcomes. Am J Epidemiol. (2009) 170:173-80. doi: 10.1093/aje/kwp101

15. IOM. Weight Gain During Pregnancy: Reexamining the Guidelines. Institute of Medicine (US) and National Research Council (US) and Committee to Reexamine IOM Pregnancy Weight Guidelines. Washington, DC (2009).

16. Gilmore LA, Redman LM. Weight gain in pregnancy and application of the 2009 IOM guidelines: toward a uniform approach. Obesity. (2015) 23:50711. doi: 10.1002/oby.20951

17. Wells G, Shea B, O'Connell D, Peterson J, Welch V, Losos M, et al. The Newcastle-Ottawa (NOS) for Assessing the Quality of Nonrandomized Studies in Meta-Analysis. Ottawa: Ottawa Hospital Research Institute. Available online at: http://www.ohri.ca/programs/clinical_epidemiology/oxford.asp

\section{AUTHOR CONTRIBUTIONS}

$\mathrm{WZ}$ and $\mathrm{XF}$ conceived, designed the study, and wrote the paper. $\mathrm{FH}$ and $\mathrm{MC}$ were involved in literature search, data collection, and analyzed the data. FZ reviewed and edited the manuscript. All authors read and approved the final manuscript.

\section{SUPPLEMENTARY MATERIAL}

The Supplementary Material for this article can be found online at: https://www.frontiersin.org/articles/10.3389/fped. 2021.674414/full\#supplementary-material

18. Higgins JPT, Green S, editors. Cochrane Handbook for Systematic Reviews of Interventions. London: The Cochrane Collaboration (2008). Available online at: https://onlinelibrary.wiley.com/doi/book/10.1002/ 9780470712184 doi: 10.1002/9780470712184

19. Pettit KE, Lacoursiere DY, Schrimmer DB, Alblewi H, Moore TR, Ramos GA. Maternal and neonatal outcomes in women with twin pregnancies with excessive gestational weight gain. J Matern Fetal Neonatal Med. (2016) 29:2182-5. doi: 10.3109/14767058.2015.10 79613

20. Wang L, Wen L, Zheng Y, Zhou W, Mei L, Li H, et al. Association between gestational weight gain and pregnancy complications or adverse delivery outcomes in chinese han dichorionic twin pregnancies: validation of the institute of medicine (IOM) (2009). Guidelines. Med Sci Monit. (2018) 24:8342-7 doi: 10.12659/MSM.911784

21. Algeri P, Pelizzoni F, Bernasconi DP, et al. Influence of weight gain, according to institute of medicine 2009 recommendation, on spontaneous preterm delivery in twin pregnancies. BMC Pregnancy Childbirth. (2018) 18:6. doi: 10.1186/s12884-017-1645-5

22. Shamshirsaz AA, Haeri S, Ravangard SF, Sangi-Haghpeykar H, Gandhi $\mathrm{M}$, Ozhand A, et al. Perinatal outcomes based on the institute of medicine guidelines for weight gain in twin pregnancies. J Matern Fetal Neonatal Med. (2014) 27:552-6. doi: 10.3109/14767058.2013.8 36177

23. Ozcan T, Bacak SJ, Zozzaro-Smith P, Li D, Sagcan S, Seligman N, et al. Assessing weight gain by the 2009 institute of medicine guidelines and perinatal outcomes in twin pregnancy. Matern Child Health J. (2017) 21:50915. doi: 10.1007/s10995-016-2134-6

24. Lutsiv O, Hulman A, Woolcott C, Beyene J, Giglia L, Armson BA, et al. Examining the provisional guidelines for weight gain in twin pregnancies: a retrospective cohort study. BMC Pregn Childbirth. (2017) 17:330. doi: 10.1186/s12884-017-1530-2

25. Lin D, Fan D, Wu S, Chen G, Li P, Ma H, et al. The effect of gestational weight gain on perinatal outcomes among Chinese twin gestations based on institute of medicine guidelines. BMC Pregn Childbirth. (2019) 19:262. doi: 10.1186/s12884-019-2411-7

26. Lal AK, Kominiarek MA. Weight gain in twin gestations: are the institute of medicine guidelines optimal for neonatal outcomes?. J Perinatol. (2015) 35:405-10. doi: 10.1038/jp.2014.237

27. Gavard JA, Artal R. Gestational weight gain and maternal and neonatal outcomes in term twin pregnancies in obese women. Twin Res Hum Genet. (2014) 17:127-33. doi: 10.1017/thg.2013.91

28. Bodnar LM, Himes KP, Abrams B, Lash TL, Parisi SM, Eckhardt CL, et al. Gestational weight gain and adverse birth outcomes in twin pregnancies. Obstet Gynecol. (2019) 134:1075-86. doi: 10.1097/AOG.0000000000003504

29. Pécheux O, Garabedian C, Drumez E, Mizrahi S, Cordiez S, Deltombe $\mathrm{S}$, et al. Maternal and neonatal outcomes according to gestational weight gain in twin pregnancies: are the institute of medicine guidelines associated with better outcomes? Eur J Obstet Gynecol Reprod Biol. (2019) 234:1904. doi: 10.1016/j.ejogrb.2019.01.010

30. Ukah UV, Bayrampour H, Sabr Y, Razaz N, Chan WS, Lim KI, et al. Association between gestational weight gain and severe 
adverse birth outcomes in washington state, Us: a populationbased retrospective cohort study, 2004-2013. PLoS Med. (2019) 16:e1003009. doi: 10.1371/journal.pmed.1003009

31. Carmichael SL, Abrams B. A critical review of the relationship between gestational weight gain and preterm delivery. Obstet Gynecol. (1997) 89(5 Pt 2):865-73. doi: 10.1016/S0029-7844(97)00047-1

32. Dietz PM, Callaghan WM, Cogswell ME, Morrow B, Ferre C, Schieve LA. Combined effects of prepregnancy body mass index and weight gain during pregnancy on the risk of preterm delivery. Epidemiology. (2006)17:1707. doi: 10.1097/01.ede.0000198470.26932.9a

33. Ellulu MS, Patimah I, Khaza'ai H, Rahmat A, Abed Y. Obesity and inflammation: the linking mechanism and the complications. Arch Med Sci. (2017) 13:851-63. doi: 10.5114/aoms.2016.58928

34. Lee H, Lee IS, Choue R. Obesity, inflammation and diet. Pediatr Gastroenterol Hepatol Nutr. (2013) 16:143-52. doi: 10.5223/pghn.2013.16.3.143

35. Hrolfsdottir L, Schalkwijk CG, Birgisdottir BE, Gunnarsdottir I, Maslova E, Granström C, et al. Maternal diet, gestational weight gain, and inflammatory markers during pregnancy. Obesity. (2016) 24:2133-9. doi: 10.1002/oby. 21617

36. Shoelson SE, Lee J, Goldfine AB. Inflammation and insulin resistance. J Clin Invest. (2006) 116:1793-801. doi: 10.1172/JCI 29069

37. Corrado F, D'Anna R, Laganà AS, Di Benedetto A. Abnormal glucose tolerance later in life in women affected by glucose intolerance during pregnancy. J Obstet Gynaecol. (2014) 34:123-6. doi: 10.3109/01443615.2013.8 41658

38. Gambioli R, Forte G, Buzzaccarini G, Unfer V, Laganà AS. Myo-Inositol as a key supporter of fertility and physiological gestation. Pharmaceuticals (Basel). (2021) 14:504. doi: 10.3390/ph14060504
39. D’Alterio MN, Sigilli M, Succu AG, Ghisu V, Laganà AS, Sorrentino F, et al. Pregnancy outcomes in women with polycystic ovarian syndrome (PCOS). Minerva Obstet Gynecol. (2021). doi: 10.23736/S2724-606X.21.04758-4. [Epub ahead of print].

40. Crawford TJ, Crowther CA, Alsweiler J, Brown J. Antenatal dietary supplementation with myo-inositol in women during pregnancy for preventing gestational diabetes. Cochr Database Syst Rev. (2015) 2015:CD011507. doi: 10.1002/14651858.CD011507.pub2

41. de Onis M., Habicht J.P. Anthropometric reference data for international use: recommendations from a world health organization expert committee. Am J Clin Nutr. (1996) 64:650-8 doi: 10.1093/ajcn/64.4.650

42. Langford A, Joshu C, Chang JJ, Myles T, Leet T. Does gestational weight gain affect the risk of adverse maternal and infant outcomes in overweight women? Matern Child Health J. (2011) 15:860-5. doi: 10.1007/s10995-008-0318-4

43. Beyerlein A, Schiessl B, Lack N, von Kries R. Optimal gestational weight gain ranges for the avoidance of adverse birth weight outcomes: a novel approach. Am J Clin Nutr. (2009) 90:1552-8. doi: 10.3945/ajcn.2009.28026

Conflict of Interest: The authors declare that the research was conducted in the absence of any commercial or financial relationships that could be construed as a potential conflict of interest.

Copyright (C) 2021 Zhong, Fan, Hu, Chen and Zeng. This is an open-access article distributed under the terms of the Creative Commons Attribution License (CC BY). The use, distribution or reproduction in other forums is permitted, provided the original author(s) and the copyright owner(s) are credited and that the original publication in this journal is cited, in accordance with accepted academic practice. No use, distribution or reproduction is permitted which does not comply with these terms. 